\title{
Laboratory Demonstration of Optimizing Optical Single Sideband Scheme to Increase Spectral Efficiency in Optical Geostationary Satellite Feeder Links
}

\author{
Ahmad Mustafa*a $^{\text {a }}$ Dirk Giggenbach ${ }^{\mathrm{a}}$, Juraj Poliak ${ }^{\mathrm{a}}$, Stephan ten Brink ${ }^{\mathrm{b}}$ \\ ${ }^{a}$ Institute of Communications and Navigation, Satellite Networks Department, \\ German Aerospace Center - DLR, 82234 Wessling, Germany \\ ${ }^{\mathrm{b}}$ Institute of Telecommunications, University of Stuttgart, 70569 Stuttgart, Germany
}

\begin{abstract}
Optical geostationary orbit (GEO) satellites are one of the means to provide high speed internet and broadband services to even remote areas on the globe. However, the optical beam, as it propagates through the atmosphere, is affected by the atmospheric index of refraction turbulence and pointing errors due to beam wander and mechanical vibrations on the platform which result in fading, hence loss of signal. We present transmit diversity as a fading mitigation technique and use wavelength division to minimize cross interference between the transmitted signals. Optical single sideband (OSSB) scheme is used to increase spectral efficiency (SE) of the system. We demonstrate a scheme where an OSSB signal is produced using commercially available optical filter with tunable bandwidth and center frequency. For a $32 \mathrm{Gbps}$ data signal modulated using amplitude shift keying (ASK), we measure the required minimum $6 \mathrm{~dB}$ and $20 \mathrm{~dB}$ bandwidths of the optical filter to be $12 \mathrm{GHz}$ and $24 \mathrm{GHz}$, respectively. Also, the offset of the filter from the carrier is found to be $-11 \mathrm{GHz}$ and $+10 \mathrm{GHz}$ to produce an error free lower and upper OSSB signal, respectively. The SE of the OSSB signal is found to be $1.34 \mathrm{bit} / \mathrm{s} / \mathrm{Hz}$. Moreover the stability of the optical filters and carrier ensure reliable signal generation making the OSSB a potential candidate to be used in future free space optical links.
\end{abstract}

Keywords: Free space optics, transmitter diversity, optical filtering, optical single sideband, bit error rate

\section{INTRODUCTION}

The ever increasing requirement of providing high speed internet services and bandwidth hungry applications to the user highlights the limitations in the currently used radio frequency communication ${ }^{1}$. Free-space optical (FSO) communications is envisaged to be used in future wireless communications due to very high optical bandwidth available, allowing much higher data rates, reduced power, size, weight and no spectrum regulation requirements. One of the means to achieve Terabits per second throughput is through optical GEO feeder links ${ }^{2,3}$. However, the optical beam, as it propagates through the atmosphere, is affected by the atmospheric index of refraction turbulence and pointing errors arising due to atmospherically induced beam wander and platform jitter ${ }^{4}$. Both result in fading, hence fluctuating received power at the satellite receiver. Transmitter diversity is an effective fading mitigation technique especially in the uplink when the size of the speckle is much larger than the receiver and the main fading contribution is due to intensity variations of the received signal $^{5,6}$. In transmitter diversity scheme multiple copies of the same signal are transmitted through uncorrelated atmospheric channel and combined in one photoreceiver. For implementation of transmitter diversity it is essential to minimize the cross-interference between transmitted signals by means of a proper division technique. Polarization division is a spectrally efficient scheme but difficult to implement and also it is limited to diversity order of two. A standard wavelength division scheme with no spectral shaping is relatively easier to implement

*ahmad.mustafa@dlr.de; phone +49 815328 3982; fax +49 815328 2844; www.dlr.de 
but it is spectrally inefficient. In order to achieve desired throughput using the available spectrum, it is required to increase the SE of the transmission system. The work presented in this paper aims at increasing the SE of the transmission system using OSSB scheme. In this work, we present the concept of a two-fold transmitter diversity system and present results of the optimization of one OSSB signal. As coherent transmission is challenging in the GEO uplink turbulent channel, we resort to using an intensity modulation with direct detection at the receiver. The spectrum of a nonreturn-to-zero (NRZ) on-off keying (OOK) ASK signal has a sideband on both sides of the carrier frequency. Since each of the sidebands has redundant information, we can filter one of them to reduce the transmitted bandwidth without losing information content. Similarly, the redundant sideband from the second signal is also filtered out. The resulting two sidebands occupy nearly the same bandwidth as one double sideband signal which means increase of SE by almost a factor of two. Here, we consider SE as the data rate that can be transmitted over a given bandwidth. The bandwidth of the OSSB signal is taken as $20 \mathrm{~dB}$ bandwidth of the optical filter.

We report results of a laboratory experiment where the OSSB signals are produced using commercially available stateof-the-art optical filters from Finisar ${ }^{7}$ which are tunable in both the center frequency and the bandwidth. We optimize the required filter bandwidth and offset from the carrier frequency to produce an OSSB signal with minimum spectral width to achieve quasi-error free bit error rate (BER) performance at a given data rate of $32 \mathrm{Gbps}$. Our measurement results show that due to the stable optical filters, lasers and other optical and electrical components in the setup, it is possible to deploy OSSB signal to increase SE of the complete system.

\section{MEASUREMENT SETUP}

\subsection{Conceptual overview of the complete scheme}

Block diagram of the concept of OSSB scheme to increase SE of the communications system in case of a two-fold transmitter diversity scheme is shown in Figure 1 and the involved components are briefly explained. Back-to-back system test for the generation of one OSSB signal was carried out to demonstrate the system aspects.

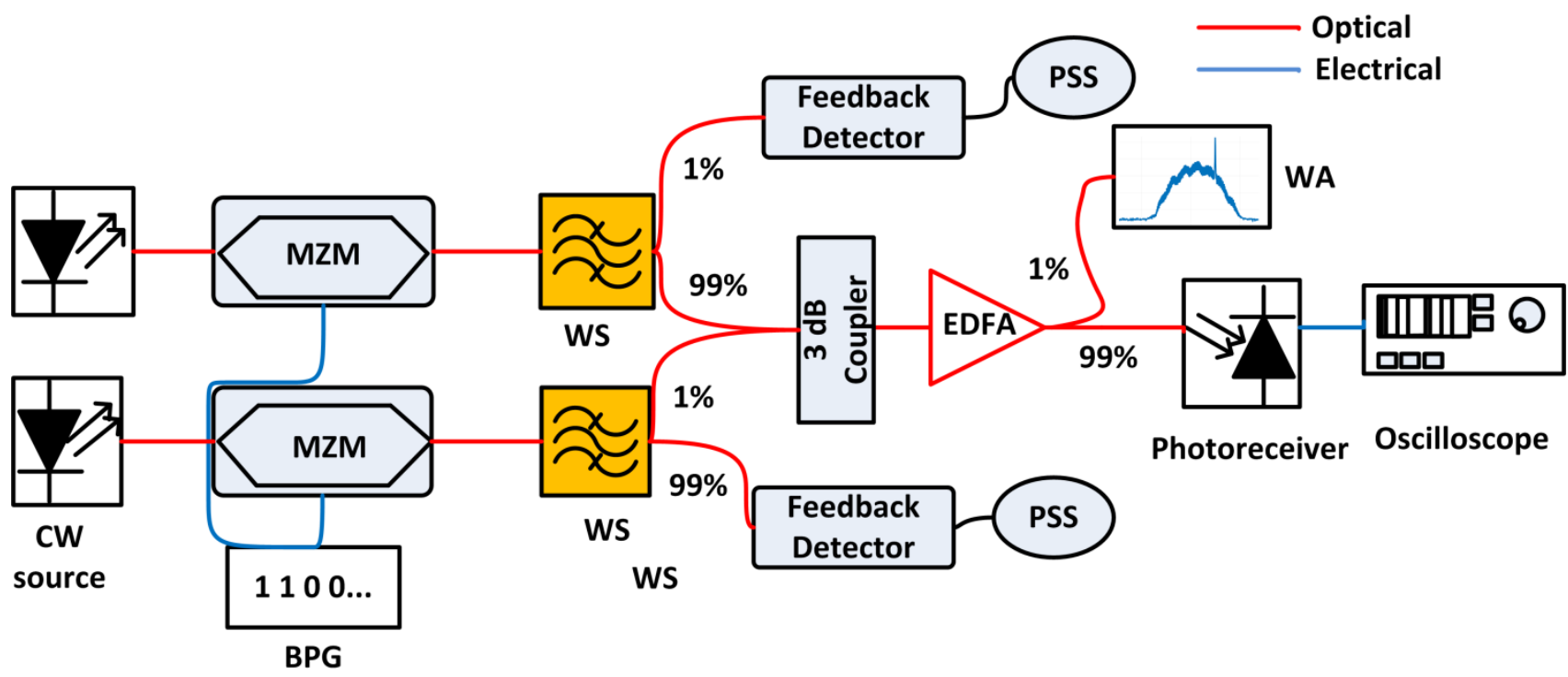

Figure 1. Block diagram of the concept of the OSSB scheme as a spectral efficient scheme

Optical source: The optical sources consist of two continuous wave (CW) lasers equipped in a chassis with polarization maintaining fiber at the output. The lasers are fully tunable over C-band and have linewidths down to $<25 \mathrm{kHz}$. This module also supports standard commands for programmable instruments (SCPI) style commands for remote control access which enable the lasers to adjust to the required power based on the feedback from the detector as explained later. 
External modulator: The two Mach-Zehnder modulators (MZM) are used for external modulation of the corresponding $\mathrm{CW}$ laser. The modulators come with automatic bias control feature to ensure proper biasing at all times. Here we use the standard intensity modulation scheme.

Bit pattern generator: The bit pattern generator (BPG) provides the data to be transmitted which is a pseudorandom bit sequence of length $2^{7}-1$. The two output channels of the BPG carry the same modulating data for the two MZMs. Moreover, it comes with an electrical bit delay feature which is helpful in synchronizing the output of the two channels as it is absolute necessary for the transmitter diversity scheme.

Optical filter: The optical filters are integrated in Finisar's waveshaper (WS) device. The waveshaper is based on high resolution liquid crystal on silicon $(\mathrm{LCoS})$ technology ${ }^{7}$ and provides full control over filter's bandwidth and center frequency. The bandwidth can be tuned from $10 \mathrm{GHz}$ to $5 \mathrm{THz}$ and the device covers the whole Cband. The functionality of remotely controlling the device using SCPI commands provides the flexibility to optimize the OSSB signal for a given data rate. The channel shape generated by the waveshaper programmable optical processor is given in eq. (1) ${ }^{7}$

$$
S(f)=\sigma \sqrt{2 \pi}\left[\operatorname{erf}\left(\frac{B / 2-\left(f-f_{0}\right)}{\sqrt{2} \sigma}\right)-\operatorname{erf}\left(\frac{-B / 2-\left(f-f_{0}\right)}{\sqrt{2} \sigma}\right)\right]
$$

where $B$ is the $6 \mathrm{~dB}$ bandwidth of the filter, $f_{0}$ is the center frequency of the filter and $\sigma$ is the standard deviation of the optical transfer function that is composed of a relatively narrow Gaussian spectrum given by eq. (2)

$$
\sigma=\frac{\mathrm{BW}_{3 \mathrm{~dB}}}{2 \sqrt{2 \ln 2}}
$$

The $3 \mathrm{~dB}$ bandwidth $\mathrm{BW}_{3 \mathrm{~dB}}$ of the optical transfer function is roughly $10 \mathrm{GHz}$, which suggests that the standard deviation is equal to $4.2466 \mathrm{GHz}$. The optical spectrum of flat-top bandpass filters created by the waveshaper is shown in Figure 2. It can be seen as the bandwidth $B$ increases, the filter shape becomes more flat at the top. It is also seen for larger $B$ values, the difference between $6 \mathrm{~dB}$ and $20 \mathrm{~dB}$ bandwidths of the corresponding filter reduces. For example, the $20 \mathrm{~dB}$ bandwidth for an optical filter with $B$ value of $12 \mathrm{GHz}$ and $100 \mathrm{GHz}$ is $24 \mathrm{GHz}$ and $111 \mathrm{GHz}$, respectively.

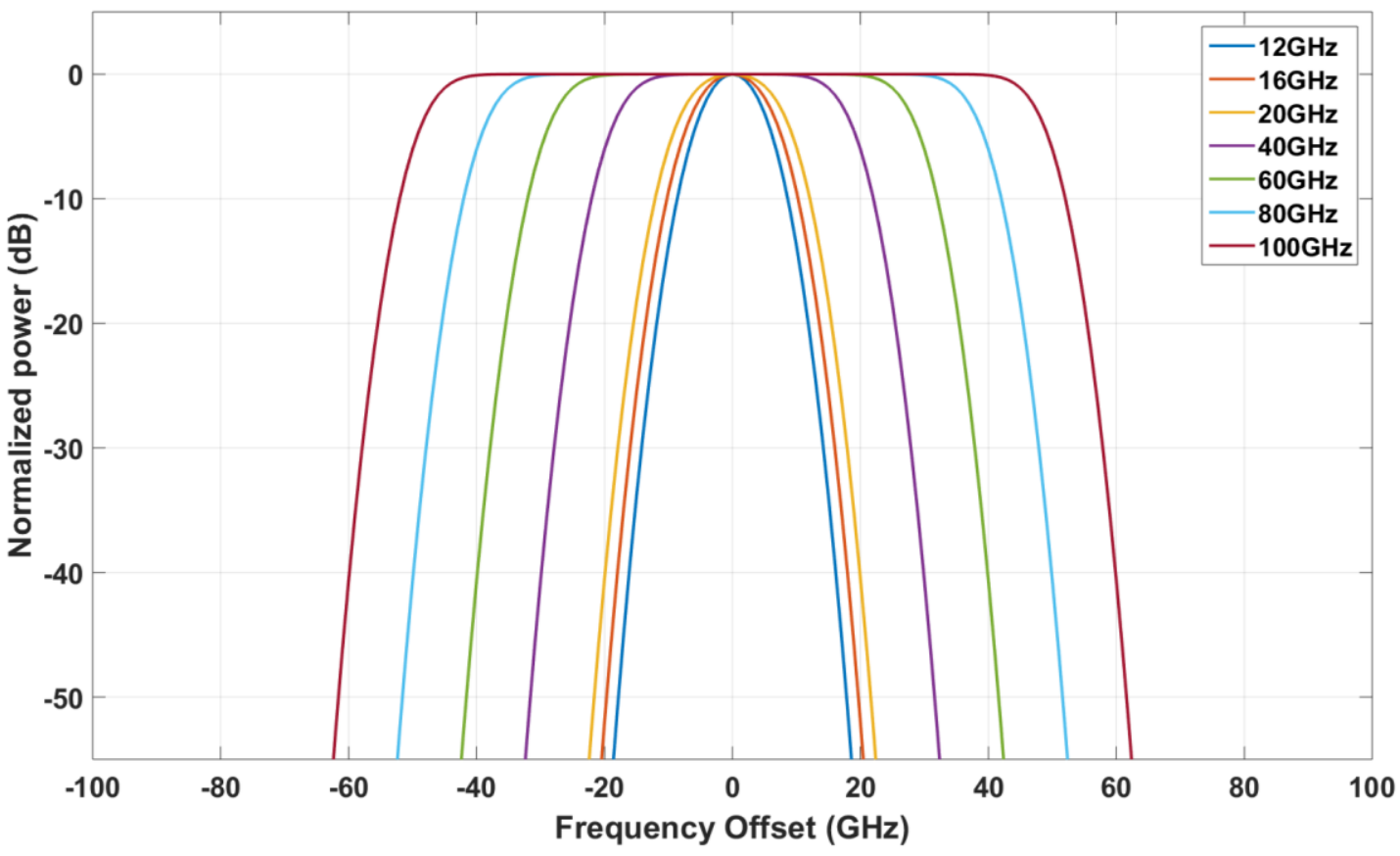

Figure 2. The filter shape, $\boldsymbol{S}(\boldsymbol{f})$, calculated for different filter bandwidths, $\boldsymbol{B}$ 
Optical splitter: One of the outputs of the $20 \mathrm{~dB}$ splitter is sent to the feedback detector and the second output goes to the $3 \mathrm{~dB}$ coupler where the signal from the second transmission branch is coupled into.

Feedback detector: The output of the feedback detector is fed to the power sampling sensor (PSS) where the DC voltage is used to calculate back the input optical power. This feedback is given to the laser which adjusts its power accordingly. This is done to keep the signal power of both the filtered signals same at all times.

Preamplifier: An erbium doped fiber amplifier (EDFA) is used for the preamplication of the incoming combined signal. The preamplifier is necessary as the receiver is not sensitive enough to detect a low powered signal when the optical filtering significantly cuts down the signal spectrum and carrier.

Photoreceiver: The linear photoreceiver has a $32 \mathrm{GHz}$ bandwidth and used in the automatic gain control mode with differential conversion gain of $500 \mathrm{~V} / \mathrm{W}$. The photoreceiver is used to make differential measurement to reduce the impact of noise. The sensitivity curve of the receiver is shown in Figure 3.

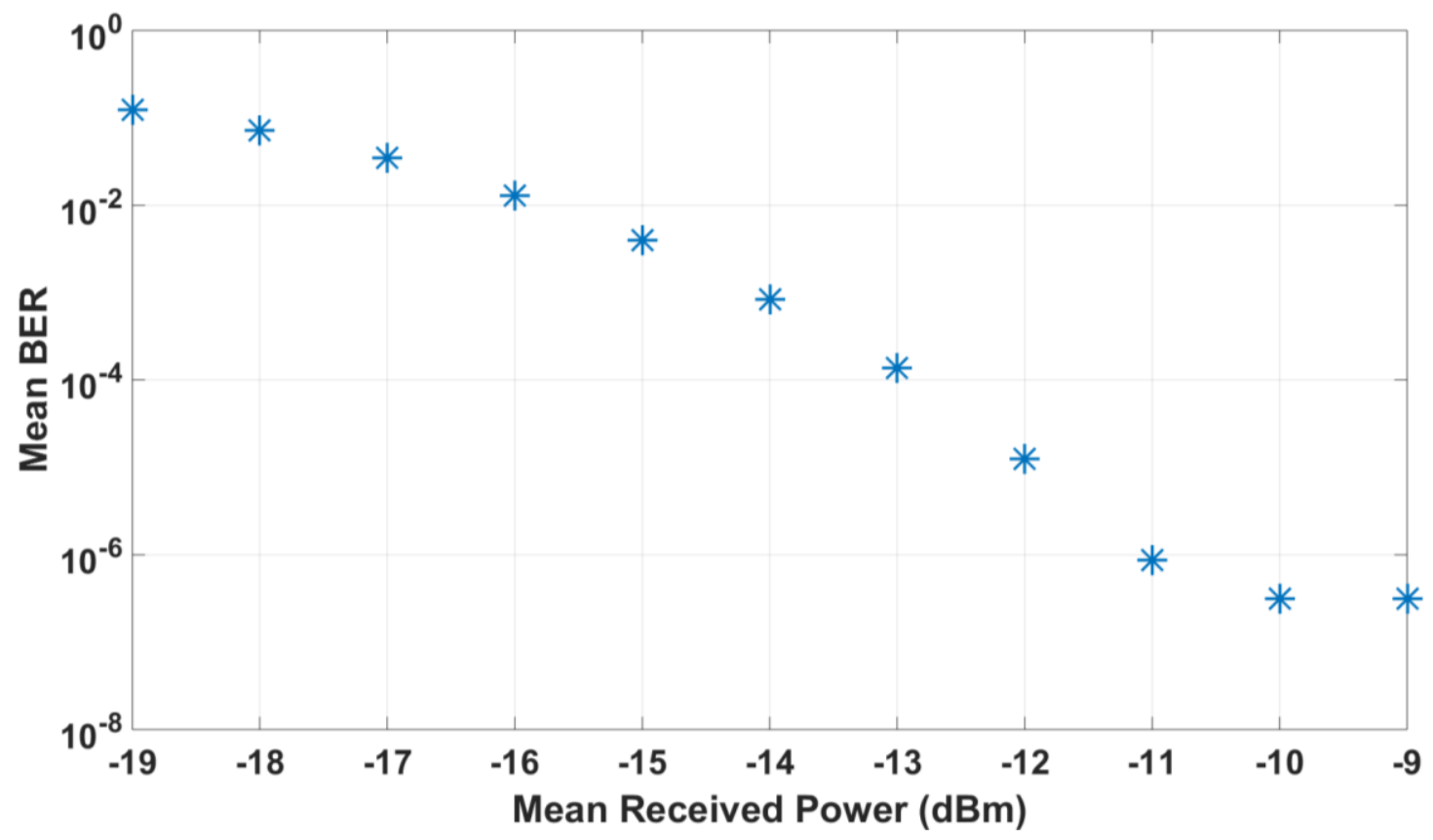

Figure 3. Receiver sensitivity curve of the Photoreceiver

Wave analyzer: The wave analyzer (WA) from Finisar is used to record the optical spectrum of the OSSB signal. The high spectral resolution of $180 \mathrm{MHz}(1.25 \mathrm{pm})$ with a fast update rate of 4 measurements per second across the entire C-band allows it to be used for modulation analysis on optical signals.

Oscilloscope: A 8 bit real-time oscilloscope with $63 \mathrm{GHz}$ analog bandwidth and sample rate of $160 \mathrm{GS} / \mathrm{s}$ is used to record the signal. The captured waveform is used to perform offline digital signal processing to obtain BER values.

\subsection{Measurement method for the optimization of one OSSB signal}

The optimization is performed on two parameters (i) minimum bandwidth required at a given data rate (ii) maximum frequency offset between the filter and the carrier to obtain lower sideband (LSB) or upper sideband (USB). The performance parameter is chosen as mean BER.

The laser source output optical power is set to its minimum value which is $6 \mathrm{dBm}$. The carrier is modulated by the PRBS7 data signal at a data rate of $32 \mathrm{Gbps}$. The modulated signal is fed to the waveshaper where initially no optical filtering is applied. The signal is only affected by the insertion loss of the waveshaper. The output 
power is taken as a benchmark and this benchmark power is kept same for the filtered signals as well. Upon filtering the signal, the power reduces which is brought up to the benchmark power by increasing the $\mathrm{CW}$ laser's power. This is achieved by taking the feedback from the feedback detector which measures the change in power between unfiltered and filtered signals using power sampling sensor. The preamplifier then amplifies the signal to $0 \mathrm{dBm}$ ensuring the photoreceiver gets enough power to detect the signal. Here our aim is to only see the effect of optical filtering on the BER. The optical spectrum of the filtered signal is observed on the high resolution optical WA. After the photoreceiver the signal is captured on a real-time oscilloscope with analog bandwidth set to $25 \mathrm{GHz}$ and sample rate of $160 \mathrm{GS} / \mathrm{s}$.

\title{
3. MEASUREMENT RESULTS
}

\subsection{Mean BER vs offset between filter and carrier}

The optical filters with varying $6 \mathrm{~dB}$ bandwidths are swept from negative to positive side of the double sideband spectrum to obtain the minimum required bandwidth and optimum filter offset from carrier at a given data rate. In Figure 4 , it can be seen that for an optical filter with $6 \mathrm{~dB}$ bandwidth of $12 \mathrm{GHz}$ and frequency offset values of $-11 \mathrm{GHz}$ and $+10 \mathrm{GHz}$, minimum BER is obtained. These two offset values give the LSB and USB signals, respectively. Beyond these values the frequency contents of the signals are so much filtered out that the signal degrades which is shown as increase in the BER values below $-11 \mathrm{GHz}$ and above $+10 \mathrm{GHz}$ for the case of optical filter with $6 \mathrm{~dB}$ bandwidth of $12 \mathrm{GHz}$. This filter has a $20 \mathrm{~dB}$ bandwidth of $24 \mathrm{GHz}$ which is considered as the minimum bandwidth required for an error free transmission of a $32 \mathrm{Gbps}$ NRZ-OOK signal. This results in a SE of $1.34 \mathrm{bit} / \mathrm{s} / \mathrm{Hz}$.

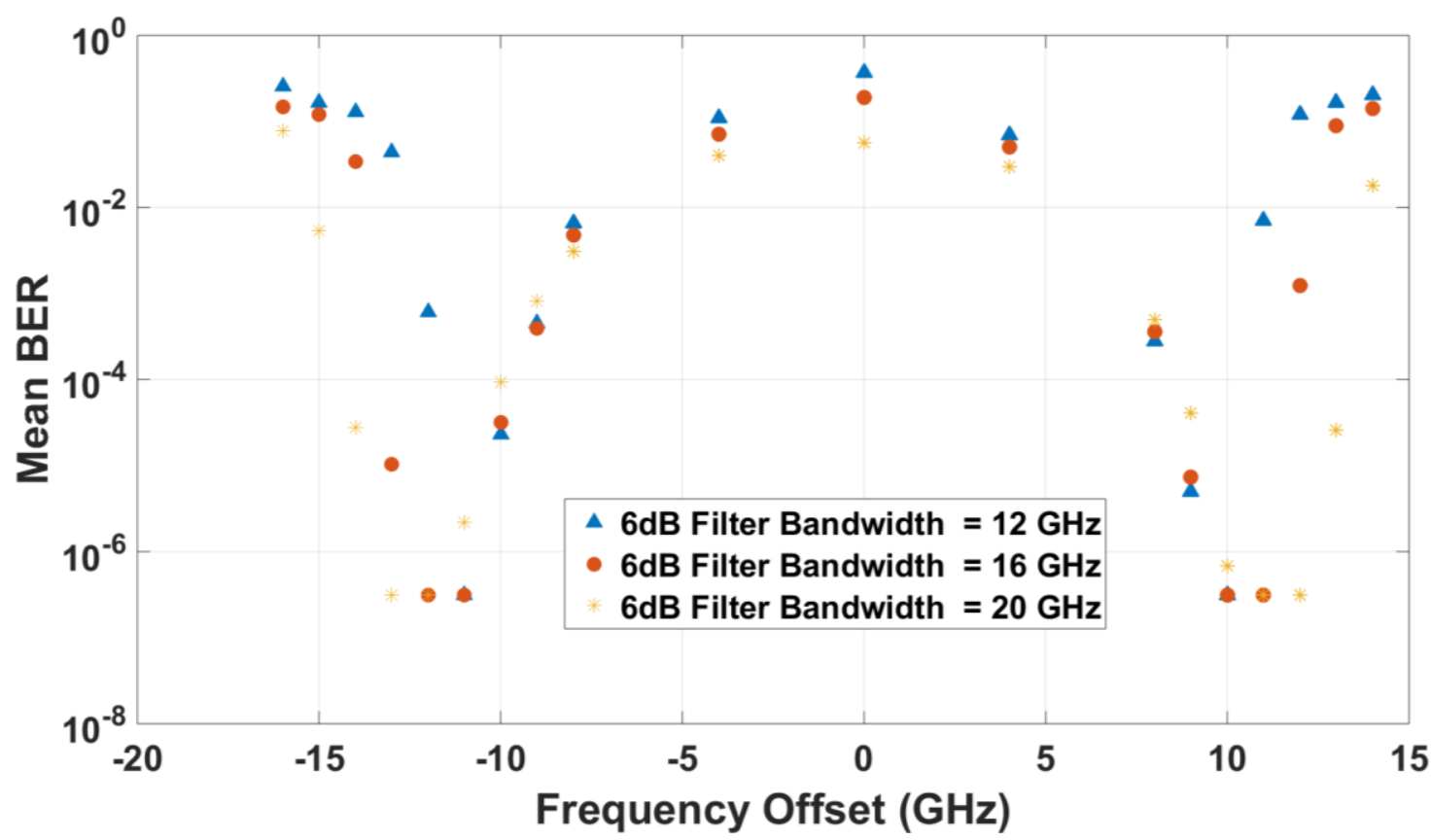

Figure 4. Mean BER Vs frequency offset between filter and carrier

\begin{abstract}
Also when the optical filter's center frequency is tuned to the carrier frequency then BER degrades as well because of insufficient frequency contents to reconstruct the signal. The frequency contents on both sides of the carrier are the same therefore the useful part of the spectrum with information content is taken as either side across the carrier. This can be seen in Figure 5 (a)-(f), along with eye diagrams and optical spectra of various filter offset settings for optical filter with $6 \mathrm{~dB}$ bandwidth of $12 \mathrm{GHz}$.
\end{abstract}




\subsection{Optical spectra and corresponding eye diagrams}

The spectrum of the unfiltered double sideband signal is shown in Figure 5(a) where it can be seen that a $32 \mathrm{Gbps}$ NRZ-OOK signal occupies $64 \mathrm{GHz}$ of bandwidth. This results in SE value of $0.5 \mathrm{bit} / \mathrm{s} / \mathrm{Hz}$. As the filter is moved from the left to the right side across the spectrum, we observe a point on the left side of the carrier where the filtered signal is error free. This is the optimum point to generate a LSB signal. Figure 5(c) shows the spectrum and the eye diagram of the LSB signal. We can see that the filter offset from the carrier is $-11 \mathrm{GHz}$. Similarly, on the right side of the carrier, we can see the optimum frequency offset point at $+10 \mathrm{GHz}$ to obtain an USB signal. The spectrum and the eye diagram of USB signal are shown in Fig 5(e).
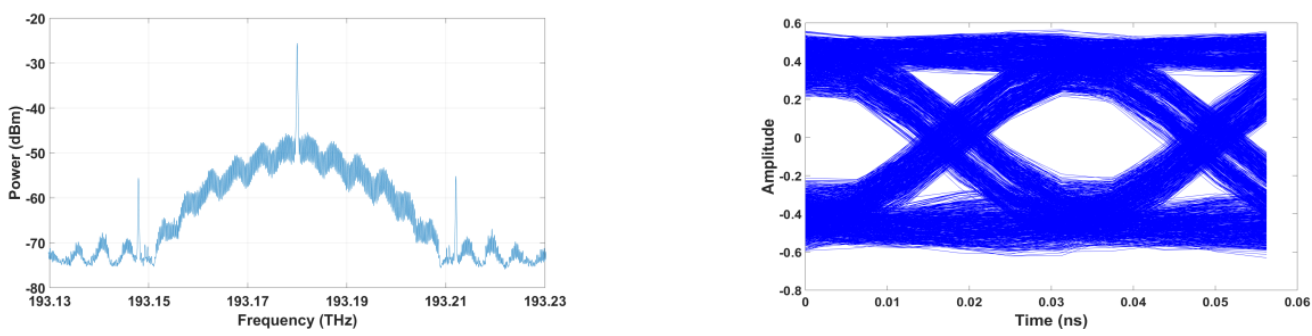

Figure 5(a). Unfiltered double sideband ASK signal $(B E R=0)$
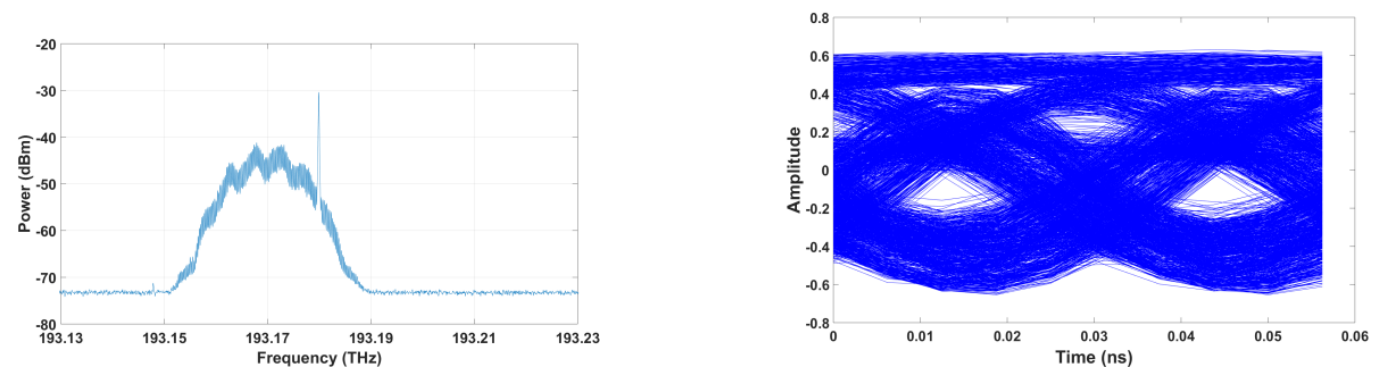

Figure $5(\mathrm{~b}) . \mathrm{BW}=12 \mathrm{GHz}$, Offset $=-14 \mathrm{GHz}(\mathrm{BER}=0.1)$
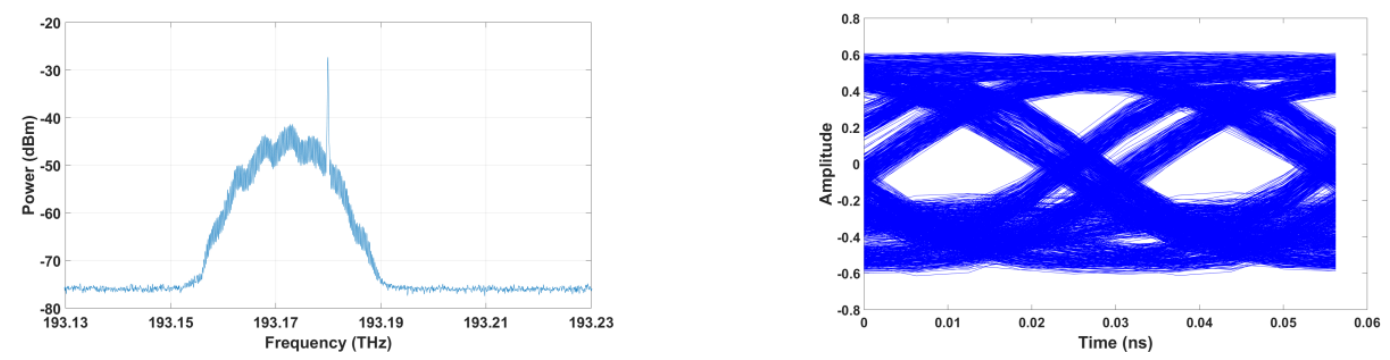

Figure 5(c). BW $=12 \mathrm{GHz}$, Offset $=-11 \mathrm{GHz}(\mathrm{BER}=0)$
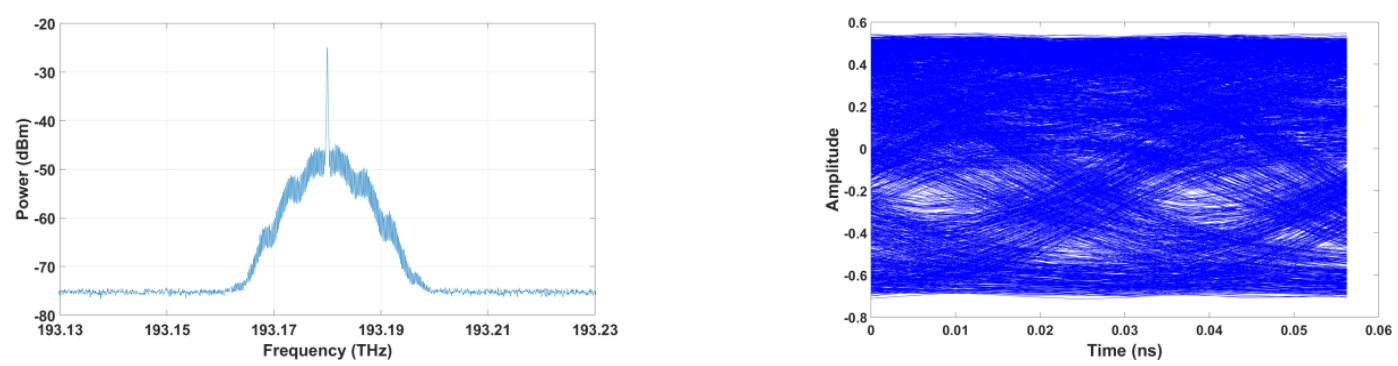

Figure 5(d). BW $=12 \mathrm{GHz}$, Offset $=0 \mathrm{GHz}(\mathrm{BER}=0.5)$ 

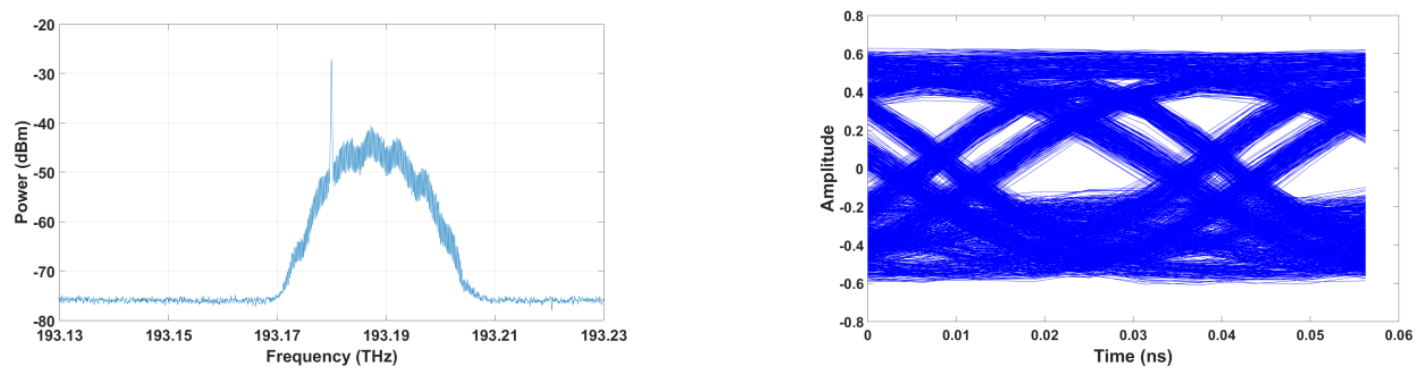

Figure $5(\mathrm{e}) . \mathrm{BW}=12 \mathrm{GHz}$, Offset $=+10 \mathrm{GHz}(\mathrm{BER}=0)$
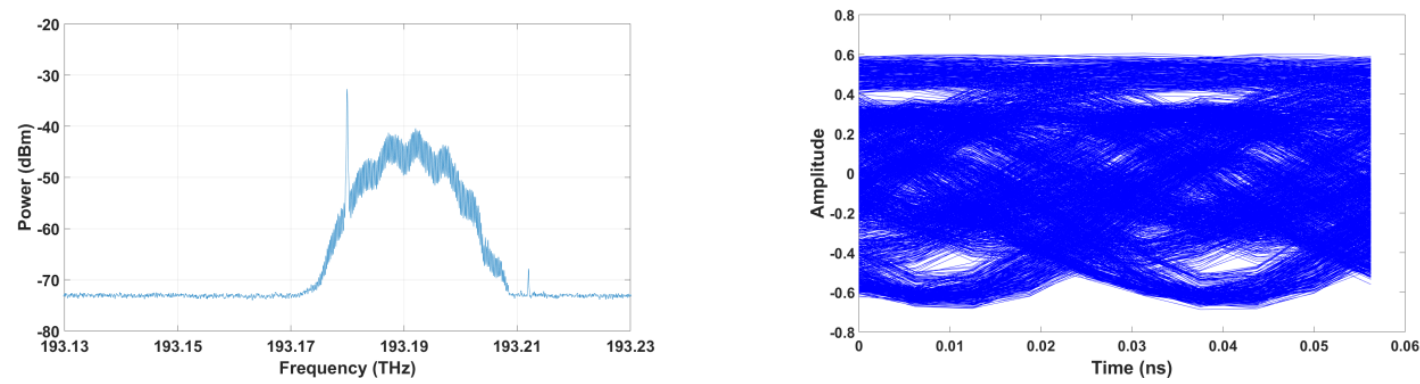

Figure $5(\mathrm{f}) . \mathrm{BW}=12 \mathrm{GHz}$, Offset $=+14 \mathrm{GHz}(\mathrm{BER}=0.3)$

\section{SUMMARY AND OUTLOOK}

An OSSB scheme for increasing SE is shown to be a promising technique due to commercially available narrow and steep optical filters. We have presented optimization of one OSSB signal considering a NRZ-OOK ASK signal at a data rate of $32 \mathrm{Gbps}$. The minimum required $6 \mathrm{~dB}$ and $20 \mathrm{~dB}$ bandwidths of the optical filter are found to be $12 \mathrm{GHz}$ and $24 \mathrm{GHz}$, respectively. The frequency offsets of the filter from the carrier are measured as $-11 \mathrm{GHz}$ and $+10 \mathrm{GHz}$ to give error free LSB and USB signals, respectively. The SE of the OSSB signal is found to be $1.34 \mathrm{bit} / \mathrm{s} / \mathrm{Hz}$ which is 2.68 times more than the SE of the unfiltered signal. As the next step, to obtain the overall SE using two-fold transmitter diversity scheme, one needs to generate the individual LSB and USB as presented in this paper and optimize the carrier separation between them to a point where interference between the two signals is tolerable to achieve the desired BER performance.

\section{ACKNOWLEDGMENT}

We deeply thank Finisar for providing the waveshaper and the wave analyzer for the measurements.

\section{REFERENCES}

[1] M. A. Khalighi and M. Uysal, "Survey on free space optical communication: A communication theory perspective," IEEE Communications Surveys Tutorials, vol. 16, no. 4, pp. 2231-2258, Fourthquarter 2014.

[2] D. Giggenbach, E. Lutz, J. Poliak, R. Mata-Calvo, and C. Fuchs, "A high-throughput satellite system for serving whole Europe with fast internet service, employing optical feeder links," in Broadband Coverage in Germany. 9th ITG Symposium. Proceedings, April 2015, pp. 1-7.

[3] J. Poliak, R. M. Calvo, and F. Rein, "Demonstration of 1.72 tbit/s optical data transmission under worst-case turbulence conditions for ground-to-geostationary satellite communications," IEEE Communications Letters, pp. 1-1, 2018.

[4] L. Andrews and R. Phillips, Laser Beam Propagation through Random Media, 2nd ed. SPIE Press, 2005. 
[5] R. Mata-Calvo, P. Becker, D. Giggenbach, F. Moll, M. Schwarzer, M. Hinz, and Z. Sodnik, "Transmitter diversity verification on artemis geostationary satellite," pp. 8971 - 8971 - 14, 2014. [Online]. Available: https://doi.org/10.1117/12.2036554

[6] A. Mustafa, D. Giggenbach, J. Poliak, A. Shrestha, R. Mata-Calvo, and C. Fuchs, "Lab implementation of 10 gbps/channel optical transmitter diversity scheme for geostationary satellite feeder links," in Photonic Networks; 16. ITG Symposium, May 2015, pp. 1-3.

[7] C. Pulikkaseril, L. A. Stewart, M. A. F. Roelens, G. W. Baxter, S. Poole, and S. Frisken, "Spectral modeling of channel band shapes in wavelength selective switches," Opt. Express, vol. 19, no. 9, pp. 8458-8470, Apr 2011. [Online]. Available: http://www.opticsexpress.org/abstract.cfm?URI=oe-19-9-8458 